\title{
CONTACT PROBLEM FOR BONDED NONHOMOGENEOUS MATERIALS UNDER SHEAR LOADING
}

\author{
B. M. SINGH, J. ROKNE, R. S. DHALIWAL, and J. VRBIK
}

Received 24 November 2002

\begin{abstract}
The present paper examines the contact problem related to shear punch through a rigid strip bonded to a nonhomogeneous medium. The nonhomogeneous medium is bonded to another nonhomogeneous medium. The strip is perpendicular to the $y$-axis and parallel to the $x$-axis. It is assumed that there is perfect bonding at the common plane surface of two nonhomogeneous media. Using Fourier cosine transforms, the solution of the problem is reduced to dual integral equations involving trigonometric cosine functions. Later on, the solution of the dual integral equations is transformed into the solution of a system of two simultaneous Fredholm integral equations of the second kind. Solving numerically the Fredholm integral equations of the second kind, the numerical results of resultant contact shear are obtained and graphically displayed to demonstrate the effect of nonhomogeneity of the elastic material.
\end{abstract}

2000 Mathematics Subject Classification: 45B05, 74B05, 45F10, 33C10.

1. Introduction. In practical applications, the material nonhomogeneity becomes an important factor to be considered, particularly, in the certain class of problems such as the foundation and contact problems in soil mechanics and wave propagation problems in the mechanics. In solid mechanics, many of the engineering materials, such as composites and large variety of bonded material and structural components, are generally modeled as nonhomogeneous continua. An example of a material with these properties is shale-sandstone. There are some important applications in ceramic coating of metal substrates and in metal-ceramic composites with graded properties. The advantages of functionally gradient materials are that of the materials which can resist high temperatures effectively and the thermal stresses in the material can be reduced significantly. Therefore, those materials cannot break easily and they have important applications in engineering.

Related to the present work is the solution of the mixed boundary value problems in nonhomogeneous medium discussed by Singh [4], Dhaliwal and Singh [2], and Delale and Erdogan [1]. In these papers, the nonhomogeneity vary in the two directions of the coordinate axes. It is important to mention that Erdogan [3] discussed crack problems under antiplane shear loading in bonded materials, where shear moduli vary exponentially as a function of $x$. Even though, no systematic study of the problem discussed in this paper 
appears to have been made, it is reasonable to expect that, in nonhomogeneous materials with continuous and continuously differentiable elastic constants, the nature of stress singularity at the punch corner would be identical to that of a homogeneous medium.

In this paper, we consider the finite antiplane shear punch on the nonhomogeneous medium $0<x<\infty$ and $0<y<\infty$. The medium has shear modulus $\mu(x, y)=G_{1} e^{\alpha_{1} x+\beta y}$ and the medium is bonded by another nonhomogeneous medium $-\infty<x<0$ with shear modulus $\mu(x)=G_{2} e^{\alpha_{2} x}$. The formulation of the problem leads to the solution of dual integral equations with trigonometric cosine function. Finally, the solution of the problem is reduced to the solution of two simultaneous system of Fredholm integral equations of the second kind in two unknown functions. The expressions for shear stress and resultant shear force under the punch are obtained. The simultaneous system of Fredholm integral equations is numerically solved and numerical values for resultant shear force under the punch and shear stress are graphed to demonstrate the effect of nonhomogeneity of the elastic material.

This paper also contains a novel treatment of contact problems involving a nonhomogeneous space and has practical value in soil mechanics and mining engineering. This paper has importance for the study of some materials that are nonhomogeneous in the $x$ and $y$ directions.

2. Solution of equilibrium equation in rectangular Cartesian coordinates. We consider a solid under shear such that the displacement field is given by

$$
u_{x}(x, y, z)=0, \quad u_{y}(x, y, z)=0, \quad u_{z}(x, y, z)=w(x)
$$

The only nonzero stress components are given by

$$
\sigma_{x z}(x, y)=\mu \frac{\partial w}{\partial x}, \quad \sigma_{y z}(x, y)=\mu \frac{\partial w}{\partial y}
$$

where $\mu$, the modulus of rigidity of the material, is assumed to be continuous and twice differentiable function of $x$ and $y$. In this case, the equation of equilibrium of an isotropic elastic material in the absence of body force is given by

$$
\frac{\partial}{\partial x}\left(\mu \frac{\partial w}{\partial x}\right)+\frac{\partial}{\partial y}\left(\mu \frac{\partial w}{\partial y}\right)=0 .
$$

If we take

$$
w(x, y, z)=\frac{W(x, y)}{\sqrt{\mu(x, y)}},
$$


then making use of (2.4), (2.3) takes the following form:

$$
\mu \Delta^{2} W+\frac{1}{2}\left[\frac{1}{2 \mu}\left\{\left(\frac{\partial \mu}{\partial x}\right)^{2}+\left(\frac{\partial \mu}{\partial y}\right)^{2}\right\}-\frac{\partial^{2} \mu}{\partial x^{2}}-\frac{\partial^{2} \mu}{\partial y^{2}}\right] W=0
$$

where, in Cartesian coordinates, the Laplacian operator $\Delta^{2}$ takes the form

$$
\Delta^{2}=\frac{\partial^{2}}{\partial x^{2}}+\frac{\partial^{2}}{\partial y^{2}}
$$

Now assume that $W$ and $\mu$ can be taken in the form

$$
W(x, y)=X(x) Y(y), \quad \mu=\mu_{0} p(x) q(y)
$$

where $\mu_{0}$ is a constant. Substituting (2.7) into (2.5) for $W$ and $\mu$, we find that $X$ and $Y$ satisfy the following differential equations:

$$
\begin{gathered}
X_{x x}+\left[k^{2}+\frac{1}{4}\left(\frac{p_{x}}{p}\right)^{2}-\left(\frac{p_{x x}}{p}\right)\right] X=0, \\
Y_{y y}+\left[-k^{2}+\frac{1}{4}\left(\frac{q_{y}}{q}\right)^{2}-\left(\frac{q_{y y}}{q}\right)\right] Y=0,
\end{gathered}
$$

where

$$
p_{x}=\frac{\partial p}{\partial x}, \quad p_{x x}=\frac{\partial^{2} p}{\partial x^{2}}
$$

and $k$ is a separating constant. If we assume that

$$
\frac{p_{x x}}{2 p}-\frac{1}{4}\left(\frac{1}{p} \frac{d p}{d x}\right)^{2}=a_{0}, \quad \frac{q_{y y}}{2 p}-\frac{1}{4}\left(\frac{1}{q} \frac{d q}{d y}\right)^{2}=b_{0},
$$

where $a_{0}$ and $b_{0}$ are constants, we find that (2.8) can be written in the form

$$
\begin{aligned}
& X_{x x}+\left(k^{2}-a_{0}\right) X=0, \\
& Y_{y y}-\left(k^{2}+b_{0}\right) Y=0 .
\end{aligned}
$$

Making use of (2.4), (2.7), and (2.11), we find the displacement component. To find the solution of (2.11), we take the variable separation constant in the following form:

$$
\xi^{2}=k^{2}-a_{0}
$$


and write (2.11) in the following form:

$$
\begin{gathered}
X_{x x}+\xi^{2} X=0, \\
Y_{y y}-\left[\xi^{2}+a_{0}+b_{0}\right] Y=0 .
\end{gathered}
$$

The solution of (2.13) can be written as

$$
A(\xi) \cos (\xi x) e^{-\left(\xi^{2}+a_{0}+b_{0}\right)^{1 / 2} y}
$$

where $A(\xi)$ is an arbitrary constant.

An alternative solution of (2.11) can be written if we write the variable separable constant in the form

$$
\xi^{2}=k^{2}+b_{0}
$$

then the solution of (2.11) can be taken as

$$
B(\xi) \cos (\xi y) e^{-\left(\xi^{2}+a_{0}+b_{0}\right)^{1 / 2} x},
$$

where $B(\xi)$ is an arbitrary constant. In particular, we take the shear modulus in the form

$$
\mu_{1}=G_{1} e^{\alpha_{1} x+\beta y}
$$

for the region $1(0<x<\infty, 0<y<\infty)$, then comparing (2.17) with (2.7), we find that

$$
\mu_{0}=G_{1}, \quad p(x)=e^{\alpha_{1} x}, \quad q(y)=e^{\beta y} .
$$

We can easily find from (2.10) that

$$
a_{0}=\frac{\alpha_{1}^{2}}{4}, \quad b_{0}=\frac{\beta^{2}}{4} .
$$

Making use of (2.4), (2.14), (2.16) and (2.19), we find the expression for displacement component for the region $(0<x<\infty, 0<y<\infty)$ in the following form:

$$
\begin{aligned}
w^{1}(x, y)=\frac{1}{\sqrt{G_{1} e^{\alpha_{1} x+\beta y}}}\left[\int_{0}^{\infty}\{\right. & A(\xi) \cos (\xi x) e^{-\left(\xi^{2}+\left(\alpha_{1}^{2}+\beta^{2}\right) / 4\right)^{1 / 2} y} \\
& -B(\xi)\left(\frac{\sin (\xi y)}{\xi}+\frac{2}{\beta} \cos (\xi y)\right) \\
& \left.\left.\times e^{-\left(\xi^{2}+\left(\alpha_{1}^{2}+\beta^{2}\right) / 4\right)^{1 / 2} x}\right\} d \xi\right],
\end{aligned}
$$


where $A(\xi)$ and $B(\xi)$ are arbitrary functions of $\xi$. The corresponding shear stress components for the region $(0<x<\infty, 0<y<\infty)$ are as follows:

$$
\begin{aligned}
\sigma_{y z}^{1}(x, y)=-\sqrt{G_{1} e^{\alpha_{1} x+\beta y}}\left[\int_{0}^{\infty} A(\xi)\right. & \left\{\left(\xi^{2}+\frac{\alpha_{1}^{2}+\beta^{2}}{4}\right)^{1 / 2}+\frac{\beta}{2}\right\} \\
& \times \cos (\xi x) e^{-\left(\xi^{2}+\left(\alpha_{1}^{2}+\beta^{2}\right) / 4\right)^{1 / 2} y} \\
& -B(\xi) \frac{4 \xi^{2}+\beta^{2}}{2 \xi \beta} \sin (\xi y) e^{\left.-\left(\xi^{2}+\left(\alpha_{1}^{2}+\beta^{2}\right) / 4\right)^{1 / 2} x\right] d \xi} \\
\sigma_{x z}^{1}(x, y)=-\sqrt{G_{1} e^{\alpha_{1} x+\beta y}}\left[\int_{0}^{\infty}\{\right. & A(\xi)\left(\frac{\alpha_{1}}{2} \cos (\xi x)+\frac{\xi}{2} \sin (\xi x)\right) \\
& \times e^{-\left(\xi^{2}+\left(\alpha_{1}^{2}+\beta^{2}\right) / 4\right)^{1 / 2} y} \\
& -\left(\left(\xi^{2}+\frac{\alpha_{1}^{2}+\beta^{2}}{4}\right)^{1 / 2}+\frac{\alpha_{1}}{2}\right) \\
\times & B(\xi) e^{-\left(\xi^{2}+\left(\alpha_{1}^{2}+\beta^{2}\right) / 4\right)^{1 / 2} x} \\
\times & \left.\left.\left(\frac{\sin (\xi y)}{\xi}+\frac{2}{\beta} \cos (\xi y)\right)\right\} d \xi\right] .
\end{aligned}
$$

For region 2, we assume that the shear modulus is as follows:

$$
\mu_{2}=G_{2} e^{\alpha_{2} x} \quad(-\infty<x<0)
$$

For the solution of equilibrium equation (2.5) for the region 2, we can easily write the displacement and shear stress components, for the region $-\infty<x<0$, $0<y<\infty$, in the following integral form:

$$
\begin{aligned}
& w^{2}(x, y)= \frac{1}{\sqrt{G_{2} e^{\alpha_{2} x}}}\left[\int_{0}^{\infty} C(\xi) e^{\left(\xi^{2}+\left(\alpha_{2}^{2} / 4\right)\right)^{1 / 2} x} \cos (\xi y) d \xi\right], \\
& \sigma_{y z}^{2}(x, y)=-\sqrt{G_{2} e^{\alpha_{2} x}}\left[\int_{0}^{\infty} \xi C(\xi) e^{\left(\xi^{2}+\left(\alpha_{2}^{2}+\beta^{2}\right) / 4\right)^{1 / 2} x} \sin (\xi y) d \xi\right], \\
& \sigma_{x z}^{2}(x, y)=\sqrt{G_{2} e^{\alpha_{2} x}}\left[\int_{0}^{\infty}\left[\left(\xi^{2}+\frac{\alpha_{2}^{2}+\beta^{2}}{4}\right)^{1 / 2}-\frac{\alpha_{2}}{2}\right],\right. \\
&\left.\times C(\xi) e^{\left(\xi^{2}+\left(\alpha_{2}^{2} / 4\right)\right)^{1 / 2} x} \cos (\xi y) d \xi\right],
\end{aligned}
$$

where $C(\xi)$ are arbitrary functions of $\xi$.

\section{Statement of problem and derivation of Fredholm integral equation}

of the second kind. We consider the problem of the antiplane shear of a nonhomogeneous half-space $(0<x<\infty, 0<y<\infty)$ of a shear modulus $G_{1} e^{\alpha_{1} x+\beta y}$ bonded with a semi-infinite medium $-\infty<x<0, y>\infty$ of shear modulus $G_{2} e^{\alpha_{2} x}$, where $G_{1}, G_{2}, \alpha_{1}, \alpha_{2}$, and $\beta$ are real constants and $x$ and $y$ refer to Cartesian coordinates. Contact shear force is applied to the flat part 


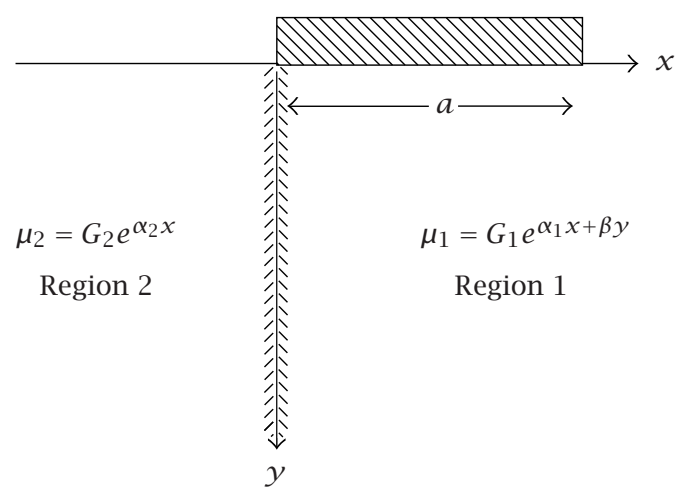

FIGURE 3.1. Contact problem for bonded nonhomogeneous materials.

of the surface $y=0,0<x<a$, through a rigid strip bounded to a nonhomogeneous medium. The geometry of the problem is shown in Figure 3.1. The boundary conditions of the problem may be taken as

$$
\begin{aligned}
w^{1}(x, 0) & =f(x), \quad 0<x<a, \\
\sigma_{y z}^{1}(x, 0) & =0, \quad a<x<\infty, \\
\sigma_{y z}^{2}(x, 0) & =0, \quad-\infty<x<0 .
\end{aligned}
$$

We have the following continuity conditions along the plane surface $x=0$ :

$$
w^{1}(0, y)=w^{2}(0, y), \quad \sigma_{x z}^{1}(0, y)=\sigma_{x z}^{2}(0, y), \quad 0<y<\infty
$$

It is required that $w^{1}(x, y)$ and $w^{2}(x, y)$ tend to zero as $\sqrt{x^{2}+y^{2}} \rightarrow \infty$ and $f(-x)=f(x) ; f(x)$ is a known function of $x$.

We find that the boundary condition (3.2) is satisfied automatically at $y=0$. Making use of (2.20), (2.22), (2.24), and (2.26), we find, from the boundary conditions (3.3), that

$$
\begin{gathered}
\int_{0}^{\infty}\left[C(\xi)+\frac{2 \sqrt{G}}{\beta} B(\xi)\right] \cos (\xi y) d \xi+\sqrt{G} \int_{0}^{\infty} \frac{B(\xi) \sin (\xi y) d \xi}{\xi} \\
=\sqrt{G} \int_{0}^{\infty} \frac{A_{1}(\xi) e^{-y e_{1}(\xi)} d \xi}{e_{1}(\xi)}, \quad 0<y<\infty, \\
\sqrt{G} \int_{0}^{\infty} e_{3}(\xi) C(\xi) \cos (\xi y) d \xi-\frac{2}{\beta} \int_{0}^{\infty} e_{4}(\xi) B(\xi) \cos (\xi y) d \xi \\
\quad-\int_{0}^{\infty} \frac{e_{4}(\xi) B(\xi) \sin (\xi y) d \xi}{\xi} \\
=-\frac{\alpha_{1}}{2} \int_{0}^{\infty} \frac{e^{-y e_{2}(\xi)} A_{1}(\xi) d \xi}{e_{1}(\xi)}, \quad 0<y<\infty,
\end{gathered}
$$


where

$$
\begin{aligned}
& e_{1}=\left[\left(\xi^{2}+\frac{\alpha_{1}^{2}+\beta^{2}}{4}\right)^{1 / 2}+\frac{\beta}{2}\right], \\
& e_{2}=\left[\left(\xi^{2}+\frac{\alpha_{1}^{2}+\beta^{2}}{4}\right)^{1 / 2}-\frac{\beta}{2}\right], \\
& e_{3}=\left[\left(\xi^{2}+\frac{\alpha_{2}^{2}}{4}\right)^{1 / 2}-\frac{\alpha_{2}}{2}\right], \\
& e_{4}=\left[\left(\xi^{2}+\frac{\alpha_{1}^{2}+\beta^{2}}{4}\right)^{1 / 2}+\frac{\alpha_{1}}{2}\right], \\
& G=\frac{G_{2}}{G_{1}}, \quad A_{1}(\xi)=e_{1} A(\xi) .
\end{aligned}
$$

Making use of Fourier cosine transforms to (3.4), we find that

$$
\begin{gathered}
\begin{aligned}
C(\xi)+\frac{2 \sqrt{G}}{\beta} B(\xi)+\frac{2 \sqrt{G}}{\pi} \int_{0}^{\infty} \frac{B(u) d u}{u^{2}-\xi^{2}} \\
=\frac{2}{\pi} \sqrt{G} \int_{0}^{\infty} \frac{A_{1}(u) d u}{\left[\xi^{2}+e_{1}^{2}(u)\right]}, \quad 0<\xi<\infty, \\
\sqrt{G} C(\xi) e_{3}(\xi)-\frac{2}{\beta} e_{4}(\xi) B(\xi)-\frac{2}{\pi} \int_{0}^{\infty} \frac{e_{4}(u) B(u) d u}{u^{2}-\xi^{2}} \\
=-\frac{\alpha_{1}}{\pi} \int_{0}^{\infty} \frac{e_{2}(u) A_{1}(u) d u}{e_{1}(u)\left[\xi^{2}+e_{1}^{2}(u)\right]}, \quad 0<\xi<\infty .
\end{aligned}
\end{gathered}
$$

Equations (3.7) have been obtained by using the following integrals:

$$
\begin{gathered}
\int_{0}^{\infty} \sin (u y) \cos (\xi y) d y=\left(\frac{u}{u^{2}-\xi^{2}}\right), \\
\int_{0}^{\infty} e^{-e_{1}(u) y} \cos (\xi y) d y=\left(\frac{e_{1}(u)}{\xi^{2}+e_{1}^{2}(u)}\right) .
\end{gathered}
$$

Eliminating $C(\xi)$ from (3.7), we get that

$$
B(\xi) e_{5}(\xi)+\int_{0}^{\infty} \frac{B(u) K_{1}(u, \xi) d u}{u^{2}-\xi^{2}}=\int_{0}^{\infty} A_{1}(u) R_{1}(u, \xi) d u, \quad 0<\xi<\infty,
$$

where

$$
\begin{aligned}
e_{5}(\xi) & =\frac{2}{\beta}\left[e_{4}(\xi)+G c_{3}(\xi)\right], \\
R_{1}(u, \xi) & =\frac{1}{\pi}\left[\frac{2 G e_{3}(\xi)}{\left[\xi^{2}+e_{1}^{2}(u)\right]}+\frac{\alpha_{1} e_{2}(u)}{e_{1}(u)\left[\xi^{2}+e_{2}^{2}(u)\right]}\right], \\
K_{1}(u, \xi) & =\frac{2}{\pi}\left[e_{4}(u)+G e_{3}(\xi)\right] .
\end{aligned}
$$


Making use of (2.20) and (2.21) in the boundary conditions (3.1), we find that

$$
\begin{gathered}
\int_{0}^{\infty} \frac{A_{1}(\xi) \cos (\xi x) d \xi}{\xi}+\int_{0}^{\infty} M_{1}(\xi) A_{1}(\xi) \cos (\xi x) d \xi \\
-\frac{2}{\beta} \int_{0}^{\infty} B(\xi) e^{x e_{6}(\xi)} d \xi=\sqrt{G_{1}} e^{\alpha_{1} x / 2} f(x), \quad 0<x<a, \\
\quad \int_{0}^{\infty} A_{1}(\xi) \cos (\xi x) d \xi=0, \quad a<x,
\end{gathered}
$$

where

$$
\begin{aligned}
& M_{1}(\xi)=\left[\frac{1}{e_{1}(\xi)}-\frac{1}{\xi}\right], \\
& e_{6}(\xi)=-\left[\xi^{2}+\frac{\alpha_{1}^{2}+\beta^{2}}{4}\right]^{1 / 2},
\end{aligned}
$$

and $A_{1}(\xi)$ is defined by (3.6).

The solution of the dual integral equations (3.11) can be written as

$$
A_{1}(\xi)=\xi(G)^{1 / 2} \int_{0}^{a} t \phi(t) J_{1}(\xi t) d t
$$

where $\phi(t)$ satisfies the following Fredholm integral equation of the second kind:

$$
\begin{aligned}
\phi(t)+ & \int_{0}^{a} \phi(u) K_{2}(u, t) d u \\
& +\frac{2}{\beta} \int_{0}^{\infty} e_{6}(\xi)\left[\frac{2}{\pi}+I_{1}\left(e_{6} t\right)+L_{1}\left(e_{6} t\right)\right] B_{1}(\xi) d \xi \\
= & -\frac{-\alpha_{1}}{2}\left[I_{1}\left(\frac{\alpha_{1} t}{2}\right)+L_{1}\left(\frac{\alpha_{1} t}{2}\right)+\frac{2}{\pi}\right], \quad 0<t<a,
\end{aligned}
$$

and

$$
\begin{gathered}
K_{2}(u, t)=u \int_{0}^{\infty} \xi^{2} M_{1}(\xi) J_{1}(\xi u) J_{1}(\xi t) d \xi \\
\sqrt{G_{1}} B_{1}(\xi)=B(\xi) .
\end{gathered}
$$

For obtaining (3.14), we have taken $f(x)=1$ and we have used the following results:

$$
\begin{aligned}
\frac{d}{d t} \int_{0}^{t} \frac{\cos (\xi x) d x}{\left(t^{2}-x^{2}\right)^{1 / 2}} & =-\left(\frac{\pi \xi}{2}\right) J_{1}(\xi t), \\
\frac{d}{d t}\left[L_{0}\left(e_{6} t\right)\right] & =e_{6}\left[\frac{2}{\pi}+L_{1}\left(t e_{6}\right)\right], \\
\frac{d}{d t} \int_{0}^{t} \frac{e^{x e_{6}} d x}{\left(t^{2}-x^{2}\right)^{1 / 2}} & =\frac{\pi e_{6}}{2}\left[\frac{2}{\pi}+I_{1}\left(e_{6} t\right)+L_{1}\left(e_{6} t\right)\right],
\end{aligned}
$$




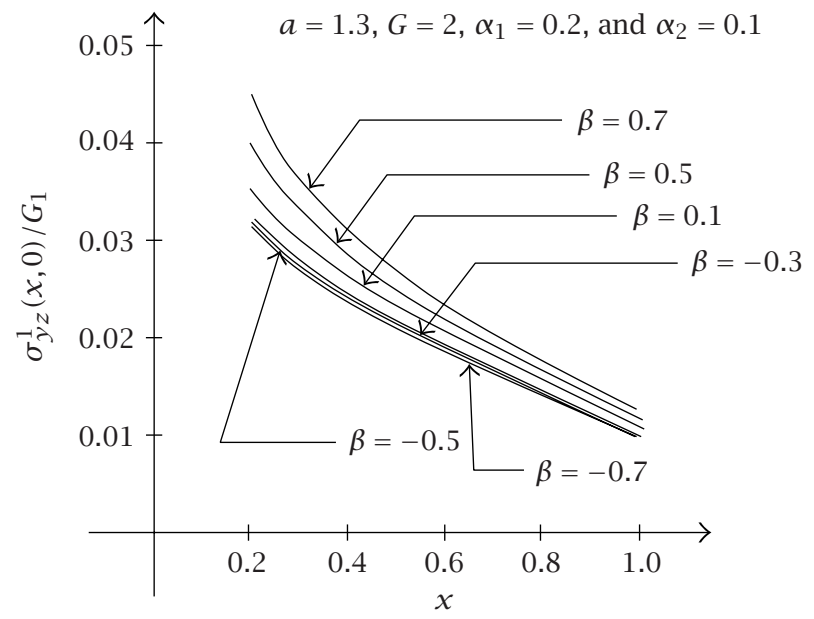

FIGURE 3.2. Variation of $\sigma_{y z}^{1}(x, 0) / G_{1}$ with $x$ for different values of $\beta=-0.7,-0.5,-0.3,0.1,0.5,0.7, a=1.3, G=2, \alpha_{1}=0.2$, and $\alpha_{2}=$ 0.1 .

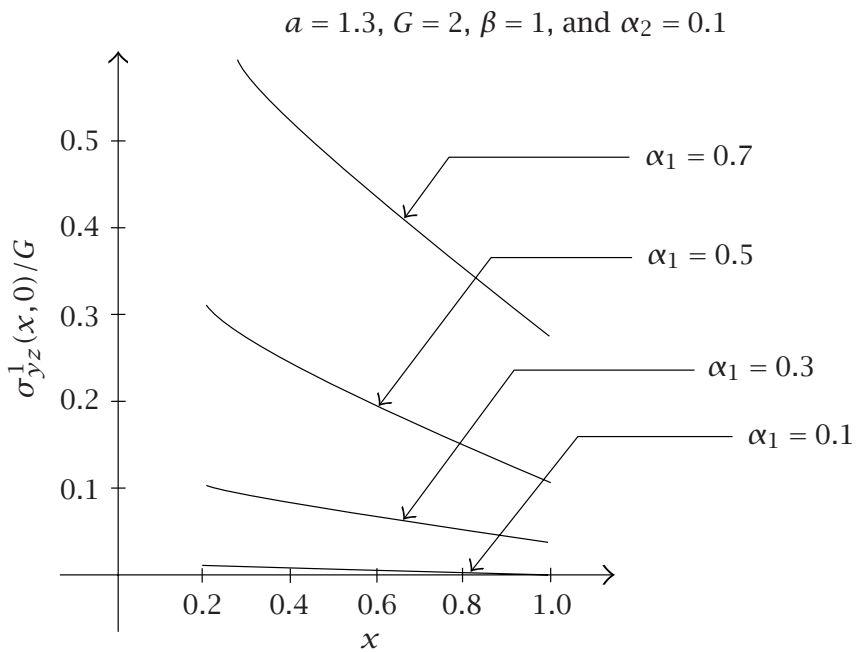

FIGURE 3.3. Variation of $\sigma_{y z}^{1}(x, 0) / G_{1}$ with $x$ for different values of $\alpha_{1}=0.1,0.3,0.5,0.7, a=1.3, G=2, \alpha_{2}=0.1$, and $\beta=1$.

where $J_{1}()$ and $I_{1}()$ are, respectively, the Bessel functions of the first kind and modified Bessel functions of the first kind, and $L_{1}()$ denotes the Struve function of the first kind of order one.

Making use of (3.13) and (3.16), (3.9) can be written in the following form:

$$
B_{1}(\xi) e_{5}(\xi)+\int_{0}^{\infty} \frac{B_{1}(u) K_{1}(u, \xi) d u}{u^{2}-\xi^{2}}=\int_{0}^{a} \phi(t) N_{1}(t, \xi) d t, \quad 0<\xi<\infty,
$$




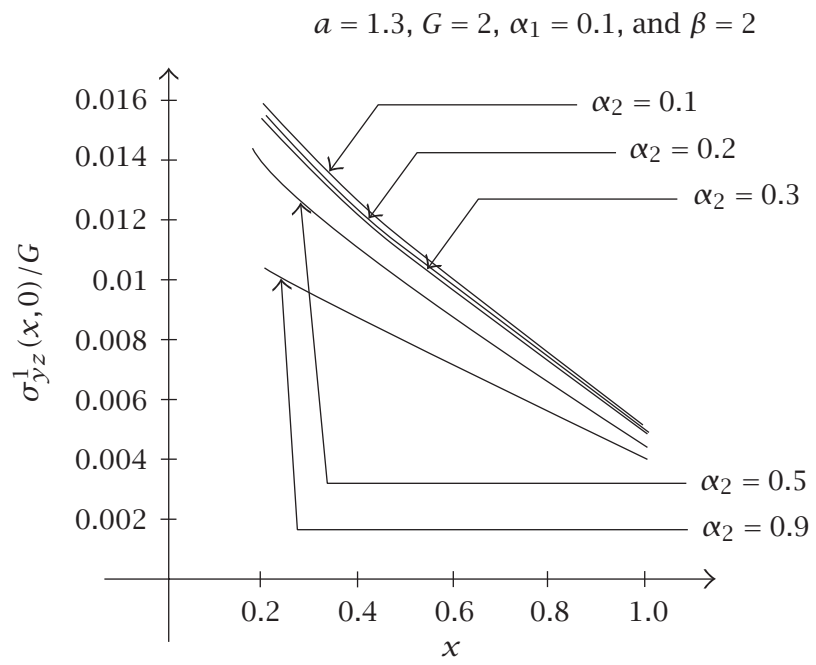

FIGURE 3.4. Variation of $\sigma_{y z}^{1}(x, 0) / G_{1}$ with $x$ for different values of $\alpha_{2}=0.1,0.3,0.5,0.7,0.9, a=1.3, G=2, \alpha_{1}=0.1$, and $\beta=2$.

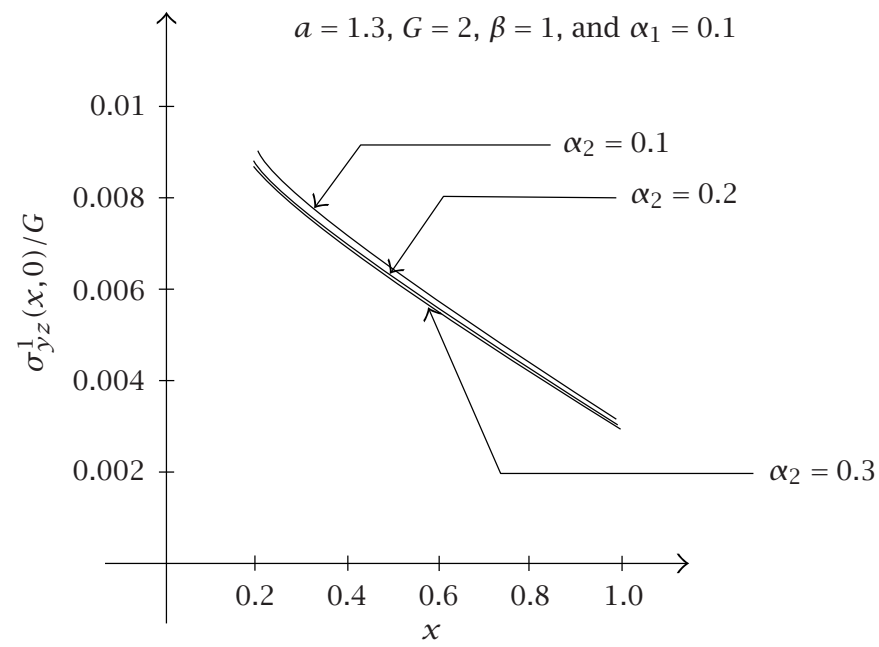

FIGURE 3.5. Variation of $\sigma_{y z}^{1}(x, 0) / G_{1}$ with $x$ for different values of $\alpha_{2}=3,5,7,9, a=1.3, G=2, \alpha_{1}=0.1$, and $\beta=2$.

where

$$
N_{1}(t, \xi)=t \int_{0}^{\infty} u J_{1}(u, t) R_{1}(u, \xi) d u
$$

Equations (3.14) and (3.18) are simultaneous Fredholm equations of the second kind. 


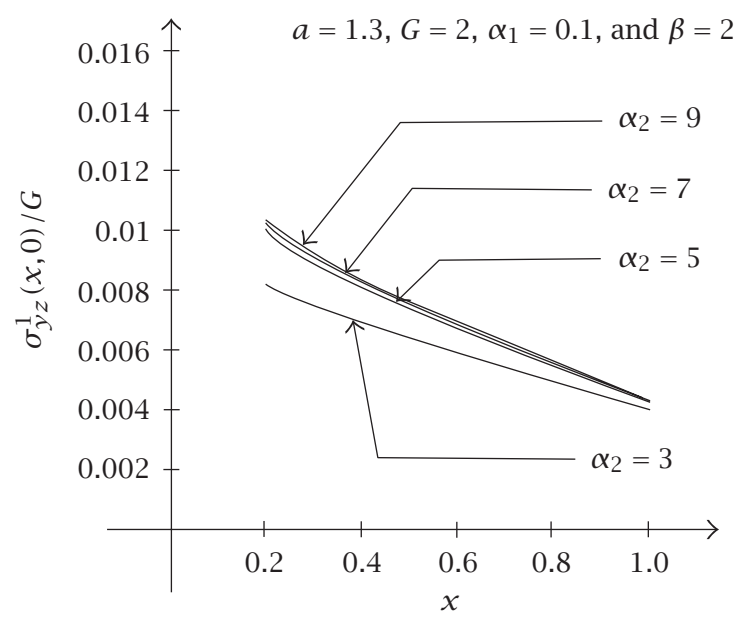

FIGURE 3.6. Variation of $\sigma_{y z}^{1}(x, 0) / G_{1}$ with $x$ for different values of $\alpha_{2}=3,5,7,9, a=1.3, G=2, \alpha_{1}=0.1$, and $\beta=2$.

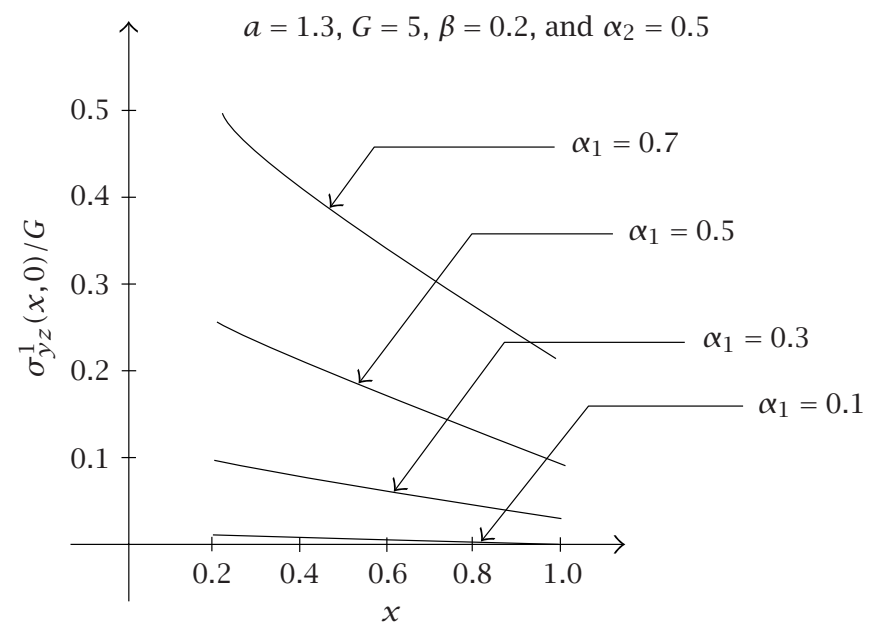

FIGURE 3.7. Variation of $\sigma_{y z}^{1}(x, 0) / G_{1}$ with $x$ for different values of $\alpha_{1}=0.1,0.3,0.5,0.7, a=1.3, G=5, \alpha_{2}=0.5$, and $\beta=0.2$.

Making use of (2.21) and (3.6), we find that

$$
\begin{aligned}
\sigma_{y z}^{1}(x, 0) & =-\sqrt{G_{1}} e^{\alpha_{1} x / 2} \int_{0}^{\infty} A_{1}(\xi) \cos (\xi x) d \xi \\
& =-\sqrt{G_{1}} e^{\alpha_{1} x / 2} \frac{d}{d x} \int_{0}^{\infty} \frac{A_{1}(\xi) \sin (\xi x) d \xi}{\xi}, \quad 0<x<a .
\end{aligned}
$$


Substituting the value of $A_{1}(\xi)$ from (3.13) into (3.20), we find that

$$
\frac{\sigma_{y z}^{1}(x, 0)}{G_{1}}=-e^{\alpha_{1} x / 2} \frac{d}{d x}\left[x \int_{x}^{a} \frac{\phi(t) d t}{\left(t^{2}-x^{2}\right)^{1 / 2}}\right], \quad 0<x<a .
$$

Solving numerically the simultaneous Fredholm integral equations (3.14) and (3.18) to get the values of $\phi(t)$ and finally using expression (3.21), we get the numerically results for $\sigma_{y z}^{1}(x, 0) / G_{1}$ which are plotted in Figures 3.2, 3.3, 3.4, 3.5, 3.6, and 3.7.

We notice from Figure 3.2 that the shear stress on the surface of the elastic material under the rigid strip decreases with $x$. Also we notice the following trend for the shear stress with the nonhomogeneity constants $\beta, \alpha_{1}$, and $\alpha_{2}$ : $\sigma_{y z}^{1}(x, 0) / G_{1}$ increases with $\beta$ in Figure 3.2, increases with $\alpha_{1}$ in Figures 3.3, 3.7, decreases with $\alpha_{2}<1$ in Figures 3.4, 3.5, and increases with $\alpha_{2}>1$ in Figure 3.6.

\section{REFERENCES}

[1] F. Delale and F. Erdogan, The crack problem for a nonhomogeneous plane, J. Appl. Mech. 50 (1983), 609-614.

[2] R. S. Dhaliwal and B. M. Singh, On the theory of elasticity of a nonhomogeneous medium, J. Elasticity 8 (1978), 211-219.

[3] F. Erdogan, The crack problem for bonded nonhomogeneous materials under antiplane shear loading, Trans. ASME J. Appl. Mech. 52 (1985), no. 4, 823-828.

[4] B. M. Singh, A note on Reissner-Sagoci problem for a nonhomogeneous solid, Z. Angew. Math. Mech. 53 (1973), 419-420.

B. M. Singh: Department of Mathematics and Statistics, University of Calgary, Calgary, Alberta, Canada T2N 1N4

J. Rokne: Department of Computer Science, University of Calgary, Calgary, Alberta, Canada T2N 1N4

E-mail address: rokne@cpsc.ucalgary.ca

R. S. Dhaliwal: Department of Mathematics and Statistics, University of Calgary, Calgary, Alberta, Canada T2N 1N4

E-mail address: dha1 i.r@shaw.ca

J. Vrbik: Department of Mathematics and Science, Brock University, St. Catherines, Ontario, Canada L2S 3A1

E-mail address: jvrbik@abacus.ac.brocku.ca 


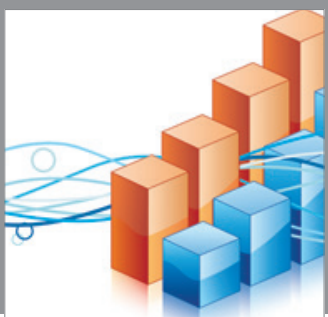

Advances in

Operations Research

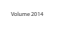

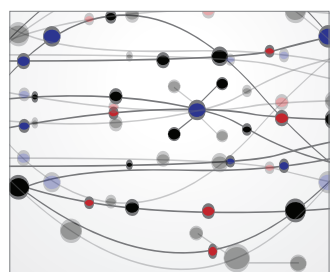

\section{The Scientific} World Journal
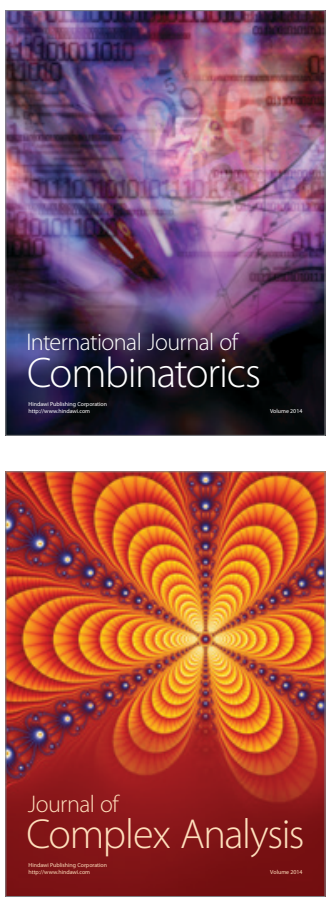

International Journal of

Mathematics and

Mathematical

Sciences
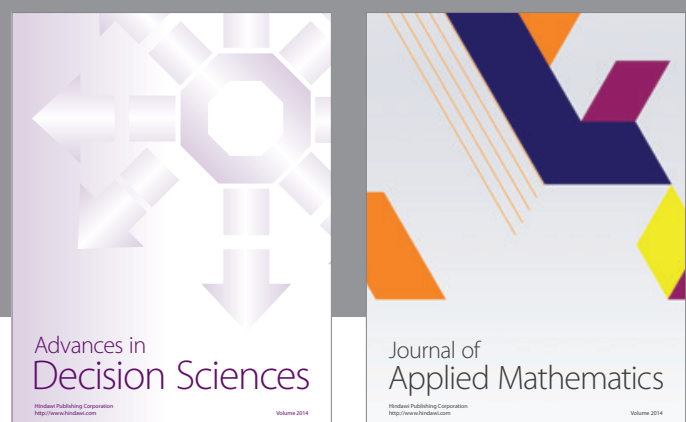

Journal of

Applied Mathematics
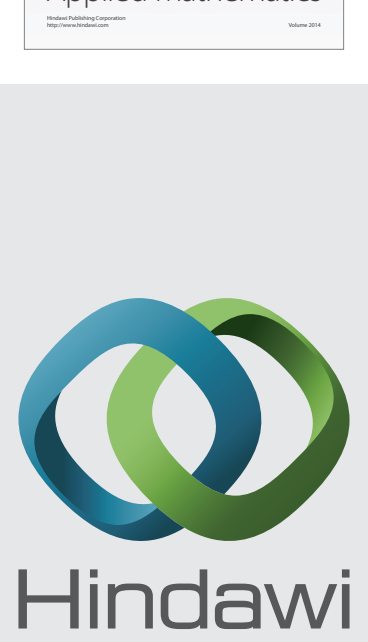

Submit your manuscripts at http://www.hindawi.com
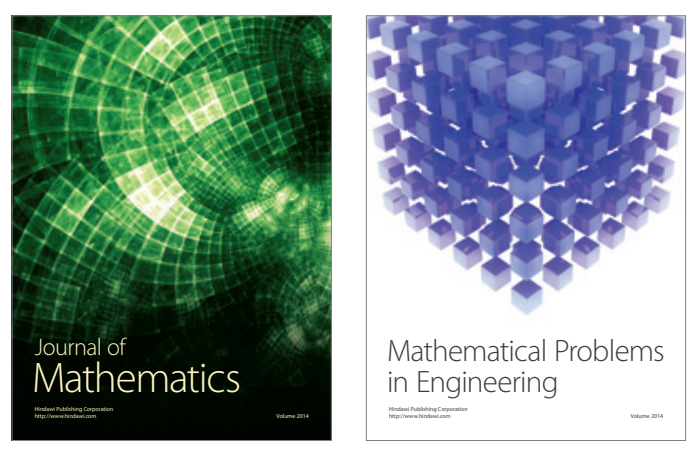

Mathematical Problems in Engineering
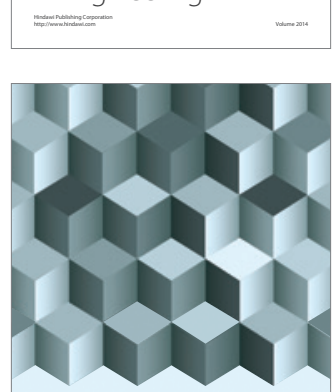

Journal of

Function Spaces
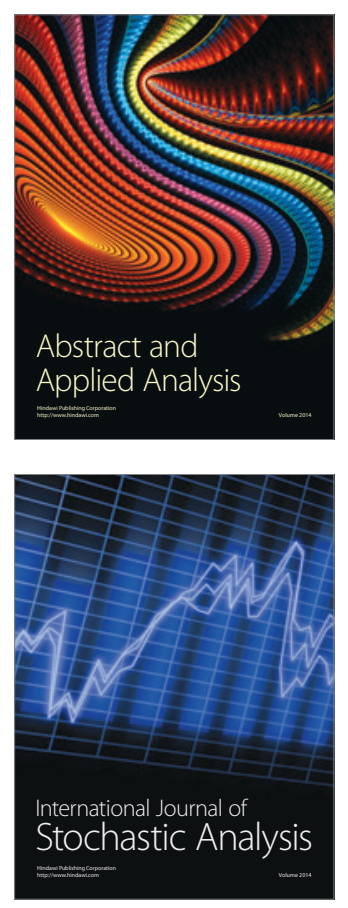

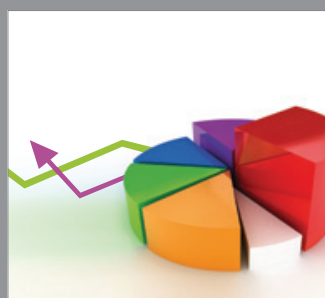

ournal of

Probability and Statistics

Promensencen
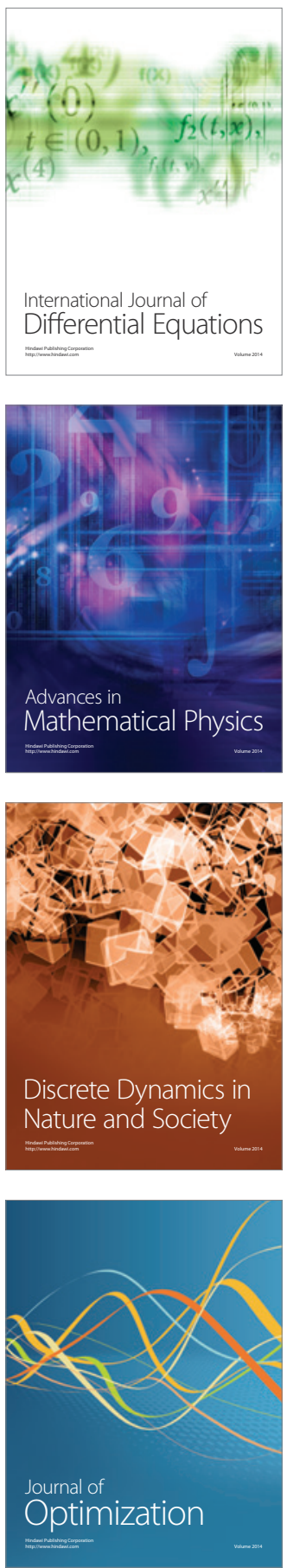